\title{
Overcoming barriers to iPS cells
}

The potential to reprogramme somatic cells into a pluripotent state to create patient-specific cells for regenerative medicine applications has attracted considerable interest in recent months. However, several important limitations - such as the tumorigenic potential of the transcription factor Myc and the use of retroviral infection - must be overcome before this technique can be broadly applied therapeutically. Three recent studies highlight approaches that may help resolve current challenges.

The first study, by Kim and colleagues, addresses the problem that ectopic expression of the transcription factor Myc causes tumorigenicity in offspring. Previous studies have shown that mouse and human somatic cells can be reprogrammed into induced pluripotent stem (iPS) cells using four transcription factors: Oct4, Sox2, Myc and Klf4. Using an Oct4-green fluorescent protein (GFP) transgenic reporter, the authors attempted to reprogramme neural stem cells (NSCs) into iPS cells using different combinations of the transcription factors. They showed that exogenous expression of Oct 4 with either Klf4 or Myc is sufficient to generate iPS cells from NSCs - the first time that an ectodermal cell type has been shown to be reprogrammed by defined transcription factor expression.

In the second paper, Huangfu and colleagues describe a different technique that also enabled generation of iPS cells without Myc. They proposed that small-molecule compounds involved in chromatin modification, previously shown to increase the efficiency of reprogramming mediated by somatic cell nuclear transfer, may also improve the efficiency of reprogramming mouse somatic cells into iPS cells. Also using an Oct4-GFP transgenic reporter, the authors tested whether DNA methyltransferase and histone

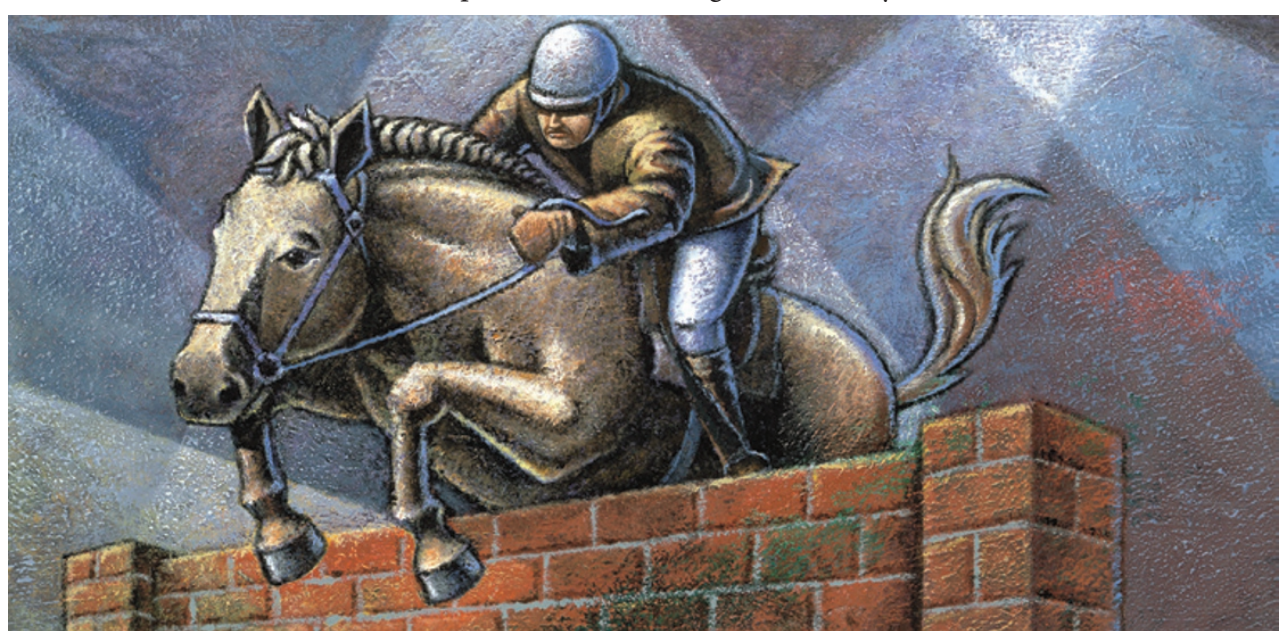

deacetylase (HDAC) inhibitors could improve reprogramming efficiency. Although all chemicals tested improved reprogramming efficiency to some extent, the HDAC inhibitor valproic acid was the most potent and enabled reprogramming with Oct4, Sox 2 and Klf4 without the introduction of Myc.

Although these two studies show that it is possible to create iPS cells without $\mathrm{Myc}$, they still require the use of retroviral transduction of the necessary transcription factors. Wernig and colleagues tackled this issue in the third study by creating somatic cells that already encode the transcription factors Oct4, Sox2, Myc and Klf4, which are inducible by administration of doxycycline (dox). Primary fibroblasts were infected with dox-inducible lentiviruses encoding the transcription factors. The resulting iPS cells were used to produce chimeric mice from which donor cells could be reprogrammed with dox with considerably greater efficiency than retroviral infection. This approach therefore provides a tool for characterizing reprogramming and performing genetic or chemical screens to enhance reprogramming of somatic cells. Bethan Hughes

ORIGINAL RESEARCH PAPERS Kim J. B. et al. Pluripotent stem cells induced from adult neural stem cells by reprogramming with two factors. Nature 29 June 2008 (doi:10.1038/nature07061) | Huangfu, D. et al. Induction of pluripotent stem cells by defining factors is greatly improved by small-molecule compounds. Nature Biotech. 26, 795-797 (2008) | Wernig, M. et al. A druginducible transgenic system for direct reprogramming of multiple somatic cell types. Nature Biotech. 1 July 2008 (doi:10.1038/nbt1483) 